ARTICLE

\title{
Semi-conducting 2D rectangles with tunable length via uniaxial living crystallization-driven self-assembly of homopolymer
}

\author{
Sanghee Yang (1) ${ }^{1}$, Sung-Yun Kang (iD ${ }^{1} \&$ Tae-Lim Choi (iD ${ }^{1 \times}$
}

\begin{abstract}
Semi-conducting two-dimensional (2D) nanoobjects, prepared by self-assembly of conjugated polymers, are promising materials for optoelectronic applications. However, no examples of self-assembled semi-conducting 2D nanosheets whose lengths and aspect ratios are controlled at the same time have been reported. Herein, we successfully prepared uniform semi-conducting $2 \mathrm{D}$ sheets using a conjugated poly(cyclopentenylene vinylene) homopolymer and its block copolymer by blending and heating. Using these as 2D seeds, living crystallization-driven self-assembly (CDSA) was achieved by adding the homopolymer as a unimer. Interestingly, unlike typical 2D CDSA examples showing radial growth, this homopolymer assembled only in one direction. Owing to this uniaxial growth, the lengths of the $2 \mathrm{D}$ nanosheets could be precisely tuned from 1.5 to $8.8 \mu \mathrm{m}$ with narrow dispersity according to the unimer-to-seed ratio. We also studied the growth kinetics of the living $2 \mathrm{D}$ CDSA and confirmed first-order kinetics. Subsequently, we prepared several 2D block comicelles (BCMs), including penta-BCMs in a one-shot method.
\end{abstract}

\footnotetext{
${ }^{1}$ Department of Chemistry, Seoul National University, Seoul 08826, Korea. ${ }^{凶}$ email: tlc@snu.ac.kr
} 
T wo-dimensional (2D) organic/polymeric nanosheets have attracted tremendous attention due to their unique properties arising from their ultrathin and flat morphology ${ }^{1-5}$. For instance, semi-conducting $2 \mathrm{D}$ materials such as graphene show optoelectronic properties that have been applied in the fields of sensors ${ }^{4}$, electronic transfer platforms $\mathrm{s}^{5,6}$, and photovoltaic cells $s^{7,8}$. To prepare such functional $2 \mathrm{D}$ nanostructures by solution process, self-assembly of semi-crystalline block copolymers (BCPs) containing a solubilizing amorphous block is one of the most powerful and facile way to achieve various morphologies including rectangles ${ }^{9}$, hexagons ${ }^{10,11}$, diamonds ${ }^{12,13}$, and squares ${ }^{14,15}$. Their core crystalline blocks can be varied from nonconjugated polymers, such as poly-(ferrocenyl dimethylsilane) $(\mathrm{PFS})^{9,10,16}$, poly(lactic acid) (PLLA) ${ }^{12,13,17}$, poly( $(\varepsilon$-caprolactone) $(\mathrm{PCL})^{11,18}$, and polyethylene $(\mathrm{PE})^{19}$, to conjugated ones, including poly(3-hexylthiophene) (P3HT) ${ }^{20,21}$, and poly (para-phenylenevinylene) $(\mathrm{PPV})^{14}$.

Since the properties of these nanomaterials are sizedependent ${ }^{22-25}$, there have been numerous efforts to develop strategies including simple blending and heating to modulate their sizes, shapes, and dimensions ${ }^{26-28}$. In particular, the most powerful and widely utilized method is crystallization-driven selfassembly (CDSA), creating uniform one-dimensional (1D) and 2D nanomaterials ${ }^{9-12,14,18}$. Taking advantage of their living polymerization-like processes, preparing even more complex block comicelles is possible ${ }^{29,30}$. However, unlike inorganic or small organic molecules ${ }^{31,32}$, semi-crystalline polymers undergo chain folding during CDSA. In the case of 2D nanosheets, this leads to radial growth of $2 \mathrm{D}$ lamellae, such that the aspect ratio can be predicted ${ }^{10,33}$, but the current technology does not allow for precise control of the length. In particular, for semiconducting $2 \mathrm{D}$ nanosheets, there is another limitation in that the strong $\pi-\pi$ interaction of the conjugated polymers lowers the solubility of the 2D nanostructures, resulting in irregular aggregation and uncontrolled self-assembly. While some problems may be solved by synthesizing BCPs containing nonconjugated shell blocks ${ }^{14}$, these insulating blocks further limit the potential of the 2D nanosheets as electronic materials.

Despite the current success in controlling 2D nanostructures, understanding the $2 \mathrm{D}$ crystallization process in solution is still in its infancy. This study is of great importance as it allows the design of new polymers, broadening the scope of $2 \mathrm{D}$ nanostructures ${ }^{34-36}$. To this end, the Manners group recently reported a growth kinetic study on the formation of $1 D$ nanofibers ${ }^{37}$. They observed that the conformational effect from the amorphous shell block of the BCPs disturbed the selfassembly process so that the kinetics were more complicated than those of analogous living polymerization and assembly of small molecules ${ }^{38-40}$. In order to obtain a clear kinetic study, controlled self-assembly of homopolymers is required to eliminate this conformational effect of BCPs. However, CDSA from homopolymers without a stabilizing shell block is extremely challenging, with only a few successes reported by the Manners group, who used a novel strategy of introducing a charged end group into homopolymers, thereby inducing electrostatic repulsion ${ }^{10,41}$. Due to these limitations, the kinetics of polymer self-assembly, especially quantitative $2 \mathrm{D}$ growth, have not been studied yet.

Regarding the formation of semi-conducting $2 \mathrm{D}$ nanosheets by self-assembly of a simple homopolymer, we recently reported two successful cases using conjugated poly(cyclopentenylene vinylene) (PCPV) homopolymers containing side chains of fluorene and bulky substituents such as neohexyl or silyl groups (Fig. 1a) ${ }^{42,43}$. We were able to control both the height and the aspect ratio of the resulting 2D nanosheets, but living CDSA was unsuccessful; instead, introducing another PCPV block as a shell, as in $\mathrm{BCP}$, allowed for the living CDSA of $1 \mathrm{D}$ nanofibers with

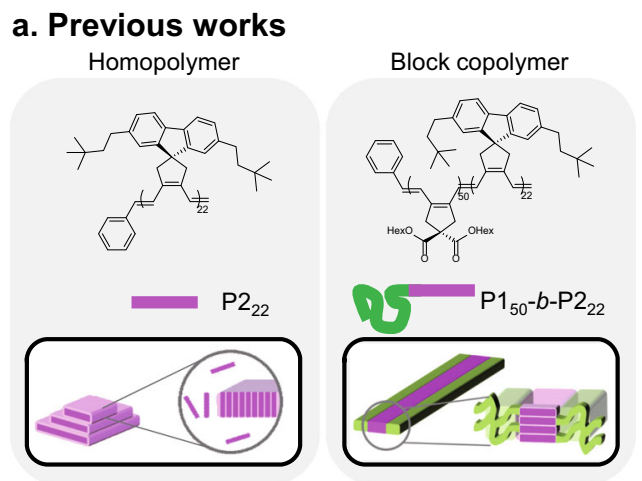

\section{b. This work}

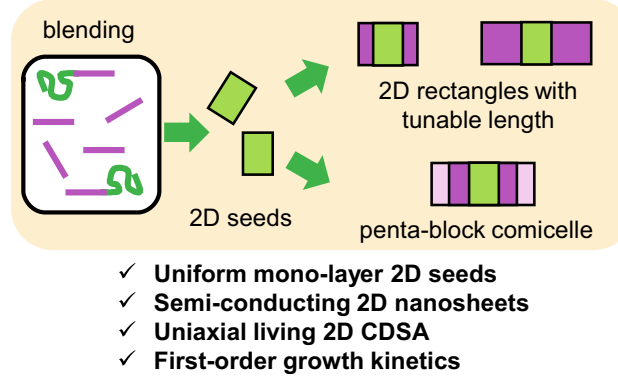

Fig. 1 Successful strategy to obtain uniform semi-conducting 2D rectangles by living CDSA. a Structures of the PCPV homopolymer and BCP and illustrations of the resulting 2D nanosheets and $1 D$ nanofibers from the PCPVs. The green color in polymer chains indicates a P1 shell block, and the pink color in polymer chains indicates a P2 core block. b Illustration of strategies for producing uniform $2 \mathrm{D}$ rectangles by living CDSA.

tunable widths and lengths (Fig. 1a) ${ }^{44}$. Inspired by earlier studies, we envisioned that blending of the $\mathrm{BCP}$ and homopolymers might provide an excellent method for precisely controlled selfassembly of semi-conducting polymers. Herein, we demonstrate the successful formation of uniform semi-conducting 2D nanosheets without stacking using a blending strategy (Fig. 1b $)^{9,45}$. From these initial 2D seeds, living 2D CDSA by a seeded-growth mechanism was possible by adding the homopolymer as a unimer. Intriguingly, the homopolymer assembled onto the 2D seeds uniaxially, resulting in the formation of length and area-controlled 2D nanorectangles with sharp edges. Based on real-time imaging of the living CDSA, kinetic studies on the uniaxial growth of $2 \mathrm{D}$ assembly revealed a first-order rate law, exactly following the living polymerization-like kinetics. Finally, we succeeded in forming complex block comicelle structures using unimers of various molecular weights.

\section{Results}

Formation of uniform 2D nanosheets by blending polymers. We previously reported that conjugated PCPV homopolymers containing because of their uniform orthorhombic crystalline arrays. For example, the semi-crystalline $\mathrm{P} 2_{22}\left(M_{\mathrm{n}}=9.01 \mathrm{kDa}\right.$ $(\nexists=1.13)$ ) homopolymer directly assembled into $2 \mathrm{D}$ rectangles in chloroform but any control of the structure was impossible because of its low solubility (Fig. 1a) ${ }^{42}$. We then expanded this moiety to $\mathrm{BCP}$ microstructures composed of identical coreforming $\mathrm{P} 2_{22}$ blocks and solubilizing $\mathrm{P} 1_{50}$ shell blocks $\left(\mathrm{P}_{50^{-}}-b\right.$ $\left.\mathrm{P} 2_{22}, M_{\mathrm{n}}=38.6 \mathrm{kDa}(\nexists=1.10)\right)$ (Fig. 1a). This modification stabilized the P2 crystalline block, enabling precise control of widths and lengths via living CDSA, but a limitation was that only 1D nanofibers were prepared by this method ${ }^{44}$. Based on these 


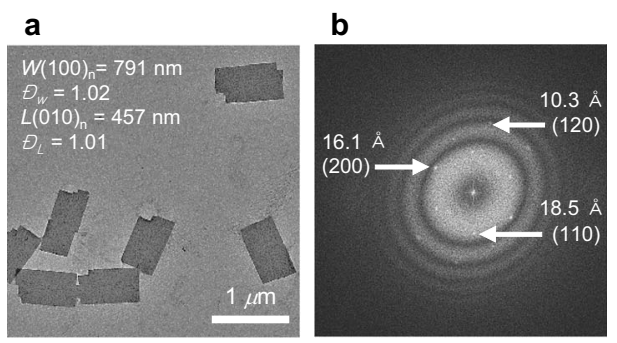

C

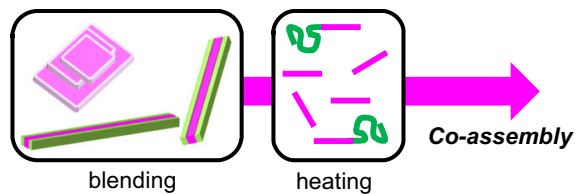

- Growth rate $(110)>(100)>(010)$

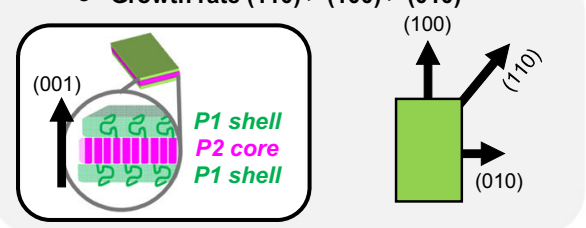

d

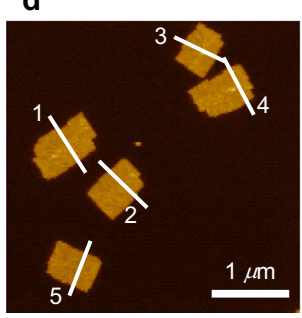

e

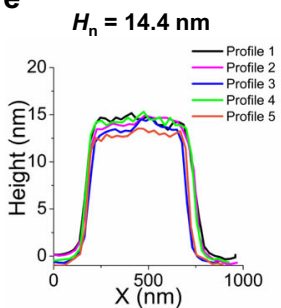

Fig. 2 Characterization of uniform monolayer 2D seeds. a TEM image of uniform 2D seeds generated by a heating and aging method using 2:1 blend ratio of $\mathrm{P} 1_{50}-b-P 2_{22}$ and $\mathrm{P} 2_{22}$ homopolymer. Numbers in the image indicate the average width $\left(W_{n}\right)$, length $\left(L_{n}\right)$, and dispersity $\left(\Theta_{w}\right.$ or $\left.\Theta_{L}\right)$. b FFT pattern from the HR-TEM image of the single $2 D$ seed showing three main $d$-spacings of $10.3,18.5$, and $16.1 \AA$ and their corresponding (hkl) planes. c Schematic representation of the formation of $2 \mathrm{D}$ seeds and their proposed detailed structures. Also included is their crystal array and observed crystal growth rates. d AFM image of 2D seeds and e height profile along the white lines shown in the AFM image, indicating the average height $\left(H_{n}\right)$.

earlier investigations, we attempted the formation of uniform $2 \mathrm{D}$ nanosheets by blending $\mathrm{P} 2_{22}$ and $\mathrm{P}_{50}-b-\mathrm{P} 2_{22}$ to achieve controlled co-assembly. Partially introduced P1 shell blocks in the blends should stabilize the main crystal array of the $\mathrm{P}_{22}$ homopolymer, overcoming the solubility issue of 2D nanorectangles in solution (Fig. 1b). To achieve this co-assembly, we used a simple heating and aging method using the blends in chloroform and screened various conditions by changing the mass ratios of the two polymers, concentration, and temperature (Supplementary Figs. 1-4).

After many optimizations, excellent co-assembly was achieved by heating a blend solution of $\mathrm{P}_{50}-b-\mathrm{P} 2_{22}$ and $\mathrm{P} 2_{22}$ at a ratio of $2: 1$ (or molar ratio of $1: 2$ ) in $0.5 \mathrm{~g} / \mathrm{L}$ chloroform at $50^{\circ} \mathrm{C}$ for $1 \mathrm{~h}$. After cooling to $25^{\circ} \mathrm{C}$ and aging for 3 days, transmission electron microscopy (TEM) imaging showed uniform monolayers of $2 \mathrm{D}$ rectangles with an aspect ratio of 1.73 and an average angle of 92.9 $9^{\circ}$, demonstrating excellent self-assembly via a self-seeding mechanism (Fig. 2a and Supplementary Fig. 5). Furthermore, the resulting $2 \mathrm{D}$ seeds had a uniform width $\left(W_{\mathrm{n}}\right)$ of $790.7( \pm 104.8)$ $\mathrm{nm}$, length $\left(L_{\mathrm{n}}\right)$ of $456.7( \pm 39.6) \mathrm{nm}$, and an area $\left(A_{\mathrm{n}}\right)$ of 0.36 $( \pm 0.063) \mu \mathrm{m}^{2}$ with very narrow length and area dispersity $(<1.02)$.
Cryogenic TEM imaging by freezing a low concentration of $0.05 \mathrm{~g} / \mathrm{L}$ in chloroform also confirmed that this $2 \mathrm{D}$ self-assembly occurred in solution and not by solvent evaporation (Supplementary Fig. 6). To gain insight into these co-assembled 2D seeds, we analyzed their electron diffraction patterns by fast Fourier transform (FFT) analysis from the high-resolution TEM (HRTEM) image (Fig. 2b and Supplementary Fig. 7). The resulting diffraction analysis showed an orthorhombic crystal lattice with main $d$-spacing values of $10.3,16.1$, and $18.5 \AA$, which were identical to those of the reported P2 homopolymer. This supports the conclusion that the blend of two polymers having common P2 cores co-crystallized into $2 \mathrm{D}$ seeds (Fig. 2c) ${ }^{42}$. Interestingly, the longer sides of the $2 \mathrm{D}$ rectangles always coincided with the direction of the (100) plane of the crystalline array. During the aging process, polymer nucleation formed small nuclei first, which then grew in both directions with slightly faster growth along the (100) plane than the (010) plane of the crystalline P2 core (cf. $(110)>(100)>(010))$ (Fig. 2c and Supplementary Fig. 8). Finally, the uniform $2 \mathrm{D}$ seeds having rectangular shapes with two distinct, well-defined crystalline surfaces were formed. In addition, The fact that the P2 core block stands upon the surface along the (001) direction of the 2D crystal lattice implies that the P1 shell blocks from the BCP would occupy the top and bottom of the $2 \mathrm{D}$ seeds, thereby suppressing the multistacking problem frequently observed in the previous single P2 assembly and making the $2 \mathrm{D}$ seeds colloidally stable ${ }^{11,42}$. This orientation also affected the height of the 2D seeds, which was measured by AFM analysis. Their average height $\left(H_{\mathrm{n}}\right)$ was $14.4 \pm 0.4 \mathrm{~nm}$, which was $3 \mathrm{~nm}$ higher than that of the previous $2 \mathrm{D}$ rectangles ${ }^{42}$ just from the P2 homopolymer without the additional P1 shell block (Fig. 2d, e, and Supplementary Fig. 9).

Uniaxial living seeded growth of homopolymers onto 2D seeds. With the uniform $2 \mathrm{D}$ seeds in hand, we investigated the possibility of CDSA via seeded growth to further control the area of 2D nanosheets. A solution of lower molecular weight P2 10 (unimer, $\left.M_{\mathrm{n}}=5.0 \mathrm{kDa}, Ð=1.15\right)$ in $10 \mathrm{~g} / \mathrm{L}$ chloroform was added to a solution of the $2 \mathrm{D}$ seeds in $0.03 \mathrm{~g} / \mathrm{L}$ chloroform $\left(A_{\mathrm{n}}\right.$ of 0.32 $\left.( \pm 0.059) \mu \mathrm{m}^{2}\right)$ with various unimer-to-seed (U/S) mass ratios from 2 to 15 . After optimizations, $\mathrm{P} 2_{10}$ successfully underwent CDSA to form uniform $2 \mathrm{D}$ rectangles, whose area increased linearly from 1.2 to $7.1 \mu \mathrm{m}^{2}(Ð<1.02)$ according to $\mathrm{U} / \mathrm{S}$ ratios after 3 weeks of aging at $-13^{\circ} \mathrm{C}$ (Figs. 3a, b, Supplementary Fig. 10). From the TEM images of the resulting $2 \mathrm{D}$ rectangles, the central 2D seeds appear darker, making them easily distinguishable from the newly formed $2 \mathrm{D}$ sheets derived from the $\mathrm{P} 2_{10}$ unimer (Fig. 3a). To our surprise, unlike other 2D platelets that grew in radial (both terminal and lateral) directions relative to the seeds, these $2 \mathrm{D}$ rectangles from the $\mathrm{P} 2_{10}$ unimer grew only in one direction along the $(010)$ plane of the $2 \mathrm{D}$ seeds. This uniaxial growth enabled us to control the $L_{\mathrm{n}}(010)$ of the resulting $2 \mathrm{D}$ rectangles from 1.5 to $8.8 \mu \mathrm{m}$ while maintaining the width $\left(W_{\mathrm{n}}\right)$ of the (100) direction (Fig. 3c and Supplementary Fig. 11, 12). Low-magnification TEM images show that the length dispersity was less than 1.03, indicating successful living 2D CDSA (Supplementary Fig. 10). Living CDSA was also qualitatively supported by DLS analysis, where the $D_{\mathrm{h}}$ values in chloroform solution gradually increased from $454 \mathrm{~nm}$ to $4.9 \mu \mathrm{m}$ as the U/S ratios increased (Fig. 2d). The height of $2 \mathrm{D}$ rectangles in the (001) direction by AFM analysis also revealed a distinct difference between the $2 \mathrm{D}$ seeds and the newly formed 2D sheets (ca. $14.4 \pm$ $0.4 \mathrm{~nm}$ versus $7.8 \pm 0.6 \mathrm{~nm}$, respectively) due to a higher DP of the P2 blocks for the seeds (Fig. 3e and Supplementary Fig. 13). To understand this unique uniaxial growth of the $2 \mathrm{D}$ rectangles along the (010) direction of the seeds as opposed to the faster 

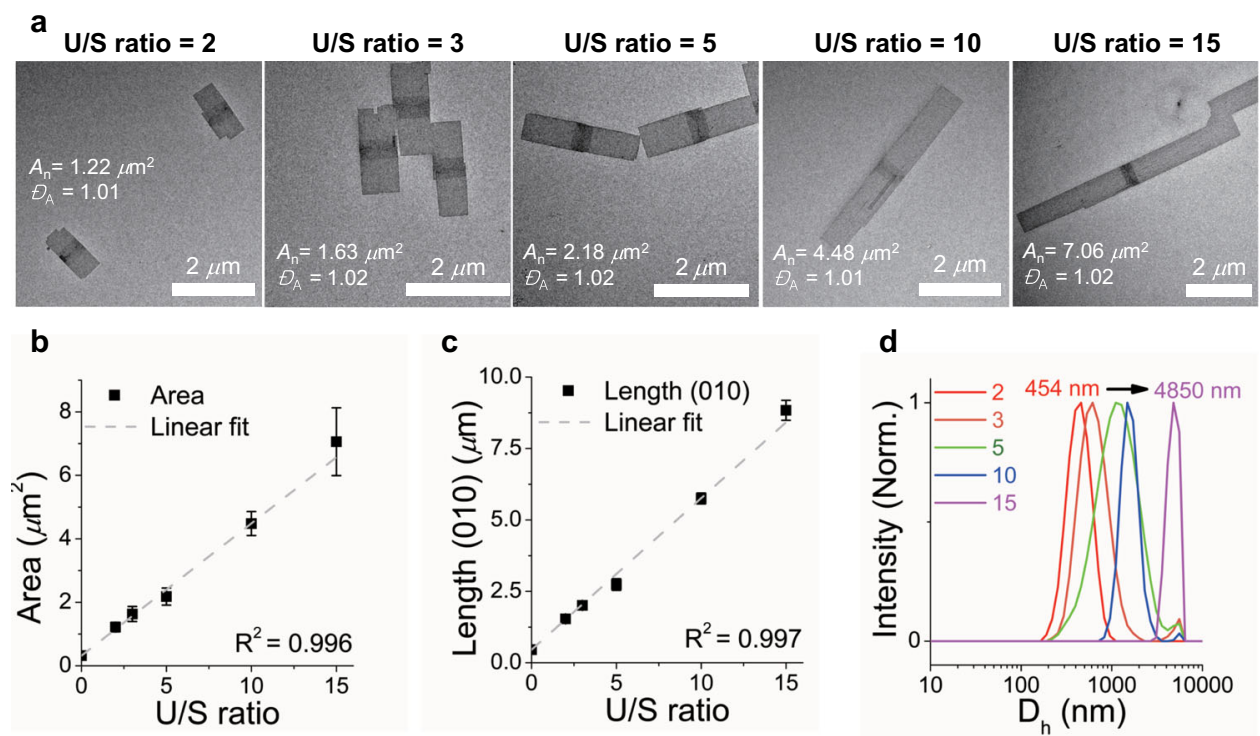

$\mathrm{U} / \mathrm{S}$ ratio $=3$

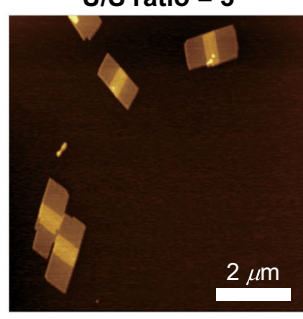

$\mathrm{U} / \mathrm{S}$ ratio $=10$
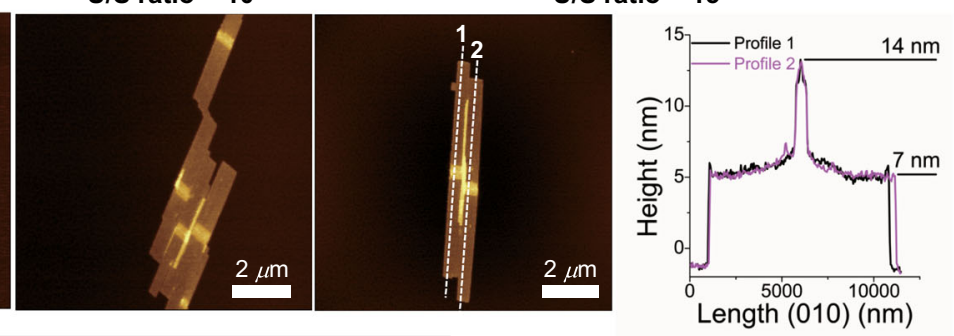

f

Uniaxial growth of $2 D$ rectangles
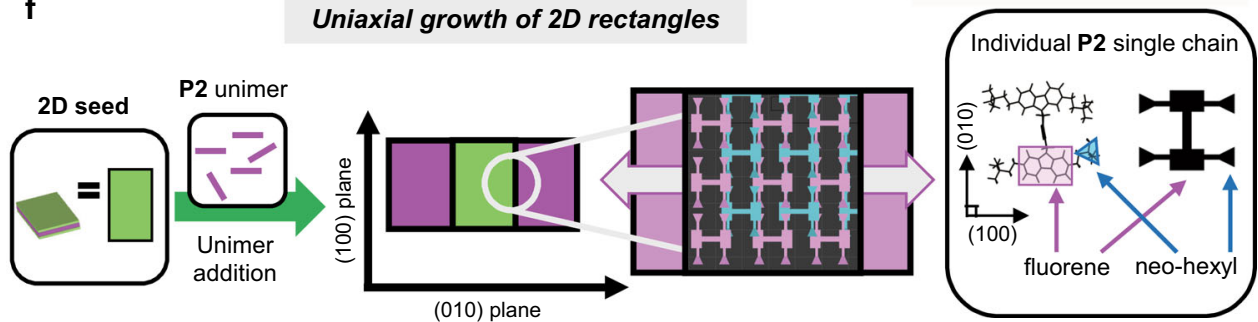

Fig. 3 Uniaxial living 2D CDSA of P2 unimer. a TEM images of length and area-controlled 2D rectangles with $\mathrm{U} / \mathrm{S}$ ratios of 2, 3, 5, 10, and 15. Numbers in the images indicate the average area $\left(A_{n}\right)$ and dispersity $\left(\Theta_{A}\right)$. Plots showing the linear dependence of $\mathbf{b}$ the area $\left(A_{n}\right)$ and $\mathbf{c}$ the average length $\left(L_{n}\right)$ in the (010) direction versus $U / S$ ratios, demonstrating living 2D CDSA. Error bars indicate standard deviations $(\sigma)$. $\mathbf{d}$ DLS profiles showing an increase in $D_{\mathrm{h}}$ from $\mathrm{U} / \mathrm{S}$ ratio of 2-15 after three weeks of aging. e AFM images of the resulting $2 \mathrm{D}$ rectangles prepared from $\mathrm{U} / \mathrm{S}$ ratios of 3,10 , and 15 . A height profile of the $2 \mathrm{D}$ rectangles with $\mathrm{U} / \mathrm{S}$ ratio of 15 , demonstrating a height difference ( $7.8 \mathrm{~nm}$ of the newly formed $2 \mathrm{D}$ sheets versus $14.4 \mathrm{~nm}$ of the original $2 \mathrm{D}$ seeds). Much darker middle blocks in TEM images and brighter middle blocks in AFM images indicate the 2D seeds. $\mathbf{f}$ Schematic representation of the living 2D CDSA via uniaxial seeded growth along the (010) direction. The 2D schematic illustration of resulting 2D rectangles is based on the interdigitating slipstack packing model of P2 homopolymer with the simplified structure in ab plane (see Supplementary Fig. 14 for detail) 42

growth in the (100) direction of the seed formation process, which might be under thermodynamic influence ${ }^{46}$, we closely examined the orientation of the orthorhombic crystal lattice of the P2 homopolymer in the $2 \mathrm{D}$ seeds (Fig. $3 \mathrm{f})^{43}$. Its (010) plane was occupied by rigid fluorene moieties of the $\mathrm{P} 2$ chains and would probably have much higher surface energy compared to the (100) plane exposing the neohexyl group (Supplementary Fig. 14). Therefore, during the elongation process, such distinct crystalline planes of the $2 \mathrm{D}$ seeds would allow the P2 unimers to kinetically crystallize onto the direction of higher surface energy, thereby leading to the preferential crystallization of unimers along the (010) direction. Similarly, in our previous finding, the 2D rectangular nanosheets from another PCPV homopolymer containing silyl groups also grew faster in the (010) direction than in the (100) direction with the $2 \mathrm{D}$ seeds having distinct crystalline surfaces ${ }^{44}$. In addition, the P2 chains containing trans alkenes exclusively seemed to exhibit a fully extended conformation without chain folding in the $2 \mathrm{D}$ arrays, thereby maximizing the selective assembly of unimers to the seeds. This defect-free CDSA might have produced the resulting $2 \mathrm{D}$ sheets having sharp edges with a nearly perfect right angle ${ }^{43}$.

Growth kinetic studies on living 2D CDSA. Most notably, this uniaxial living 2D CDSA is an excellent system for conducting kinetic studies of $2 \mathrm{D}$ assembly in solution. By real-time monitoring using TEM analysis, we conducted growth kinetics by adding $\mathrm{P} 2{ }_{10}$ homopolymer with various U/S ratios under the aforementioned conditions $(0.03 \mathrm{~g} / \mathrm{L}$ seeds in chloroform solutions at $\left.-13^{\circ} \mathrm{C}\right)$, and measuring increases in length, as described in Fig. 4a (Supplementary Table 1, Supplementary Figs. 15-19). As expected, the higher U/S ratio and concentration of unimer, 
$[\mathrm{U}]_{0}$, led to faster elongation. Interestingly, plotting $L_{\mathrm{n}}$ growth versus time fitted very well with the first-order kinetic function of $[\mathrm{U}]_{0}$, with $\mathrm{R}^{2}$ values greater than 0.991 , similar to conventional living polymerization (Table 1, Entries 1-5, Supplementary Eq. 1, Supplementary Fig. 20$)^{37}$. The rate constants (k') were also consistent within experimental errors regardless of the U/S ratio (Supplementary Table 2). Using an alternation method, the reaction order of [U], which was calculated from the initial rates by analyzing the $L_{\mathrm{n}}$ increase in the early stages, was 0.964 $( \pm 0.085)$ (Fig. 4b, Supplementary Eq. 2, Supplementary Fig. 21, Supplementary Table 3). This further confirmed the first-order kinetics of the living 2D CDSA of the $\mathrm{P} 2$ homopolymer. This is an interesting result because previous studies on living 1D CDSA of P1-b-P2 or PFS BCPs showed significant deviation from firstorder kinetics ${ }^{37,44}$. The main difference in the present study is
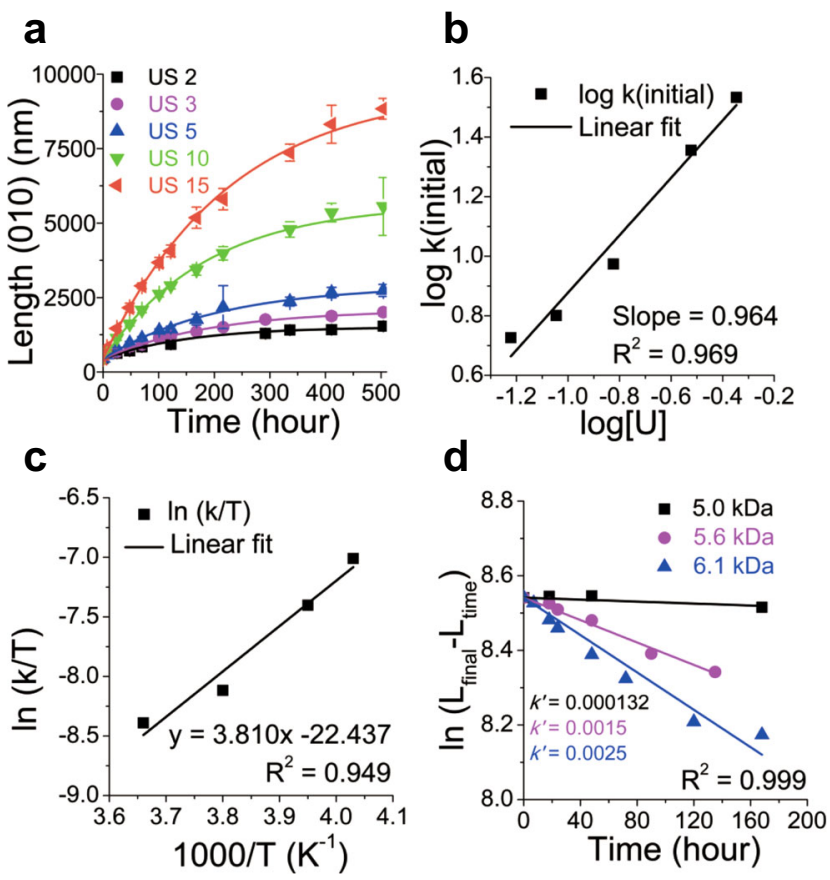

Fig. 4 Growth kinetic studies on living 2D CDSA. Plots showing a Lengths $\left(L_{n}\right)$ of $2 D$ rectangles over time with various $U / S$ ratios from 2 to 15 (monitored for 3 weeks). Error bars indicate standard deviations $(\sigma)$. b initial reaction rates versus unimer concentration, [U], to confirm first-order kinetics. c Eyring plot for the rate constants, $k^{\prime}$, extracted from seededgrowth experiments at varying temperatures. d Plot of $\ln \left(L_{\text {final }}-L\right.$ (time) $)$ versus time from the first-order growth of various unimers with different DPs at $25^{\circ} \mathrm{C}$. presumably due to the homopolymer microstructure of unimers, unlike BCPs with shell blocks in the previous cases. Therefore, the P2 homopolymer has negligible conformational effects, due to the absence of a shell block. Finally, P2, which does not even undergo chain folding, directly forms crystalline arrays, making this $2 \mathrm{D}$ CDSA analogous to ideal crystallization, similar to the living supramolecular polymerization of small organic molecules such as porphyrin derivatives ${ }^{38-40,47}$.

To explore the effect of temperature on CDSA, we again measured the rates at four different temperatures: $0,-10,-20$, and $-25^{\circ} \mathrm{C}$. After first-order fitting, the initial rate constant $k^{\prime}$ was found to increase from $3.0 \times 10^{-3}$ to $1.1 \times 10^{-2}$ as the temperature decreased from 0 to $-25^{\circ} \mathrm{C}$ (Supplementary Table 4 and Supplementary Fig. 22). These values allowed us to generate an Eyring plot to determine the activation enthalpy and entropy for the seeded-growth process, and they were negative values of $-31.7 \mathrm{~kJ} / \mathrm{mol}$ and $-384 \mathrm{~J} / \mathrm{K} \bullet \mathrm{mol}$, respectively, indicating faster growth at lower temperatures (Table 1, Entries 6-9, Fig. 4c, Supplementary Eq. 3, Supplementary Table 5, Supplementary Fig. 23) ${ }^{37}$. Furthermore, another kinetic study was performed with longer P2 unimers of DP $13(5.6 \mathrm{kDa}(\nexists=1.18))$ and 15 $(6.1 \mathrm{kDa}(\nexists=1.13))$. Since the longer $\mathrm{P} 2$ unimers showing high crystallinity easily underwent self-nucleation at low temperatures, all the kinetic experiments were performed at $25^{\circ} \mathrm{C}$ for proper comparison (Supplementary Table 6). Another first-order fitting of $[\mathrm{U}]_{0}$ provided initial rate constants $k^{\prime}$ of $1.3 \times 10^{-4}, 1.5 \times 10^{-3}$, and $2.5 \times 10^{-3}\left(\mathrm{~h}^{-1}\right)$ for P2 unimers of DP 10, 13, and 15, respectively (Table 1, Entries 10-12, Fig. 4d, Supplementary Fig. 24). This 20 times faster growth of $\mathrm{P} 2_{15}$ than $\mathrm{P} 2_{10}$ is likely due to the higher crystallinity of $\mathrm{P} 2{ }_{15}$. Similar to our previous report on $\mathrm{P}_{2}{ }^{42}$, the average height of the new $2 \mathrm{D}$ sheets increased from 7.8 to $10.4 \mathrm{~nm}$ according to the DP of P2 (Supplementary Fig. 25). With the longer P2 unimers, we were able to conduct living $2 \mathrm{D}$ CDSAs with various $\mathrm{U} / \mathrm{S}$ ratios from 1 to 10 at $25^{\circ} \mathrm{C}$, providing tunable $L_{\mathrm{n}}$ of the resulting $2 \mathrm{D}$ rectangles from 0.64 to $5.5 \mu \mathrm{m}$ for $\mathrm{P} 2_{13}$ and from 0.80 to $3.3 \mu \mathrm{m}$ for $\mathrm{P} 2_{15}$, respectively. (Supplementary Figs. 26, 27).

Formation of symmetric penta-block comicelles. Intriguingly, the uniaxial living 2D CDSA could prepare more complex multiblock comicelles (BCM) via a series of seeded growth from various P2 unimers $30,48,49$. Analogous to block copolymerization via living polymerization, the sequential addition of $\mathrm{P} 2_{10}$ and $\mathrm{P} 2_{15}$ unimers provided length and height controlled penta-BCMs along the (010) direction (unimers in $10 \mathrm{~g} / \mathrm{L}$ chloroform, U/S ratio of $3,[2 \mathrm{D}$ seeds $]=0.03 \mathrm{~g} / \mathrm{L}$ in chloroform, Fig. $5 \mathrm{a}$ ). By changing the addition order, two types of symmetric pentaBCMs, $\mathrm{A}\left(\mathrm{P} 2_{15}\right)-\mathrm{B}\left(\mathrm{P} 2_{10}\right)-\mathrm{S}($ seed)-B-A and B-A-S-A-B, were generated with uniform length and narrow dispersity (Fig. 5b,

Table 1 Kinetic data for 2D CDSA experiments with different conditions: rate constants ( $\left.k^{\prime}\right)$ with standard errors and $\mathbf{R}^{2}$.

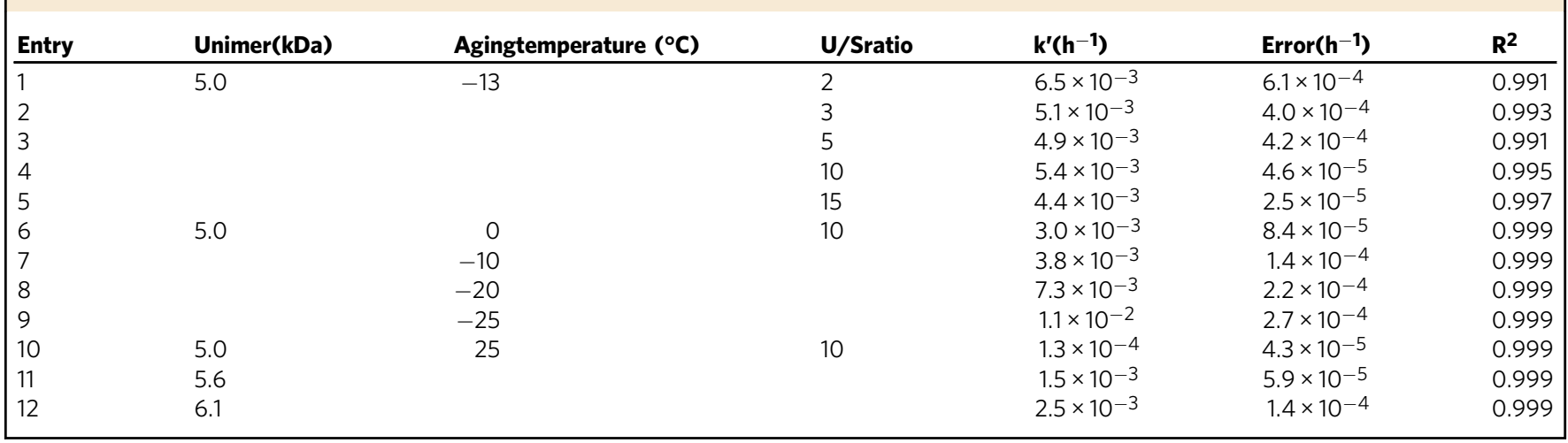


a

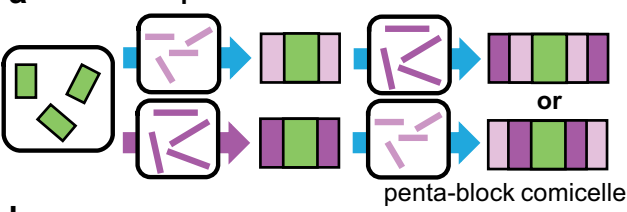

b

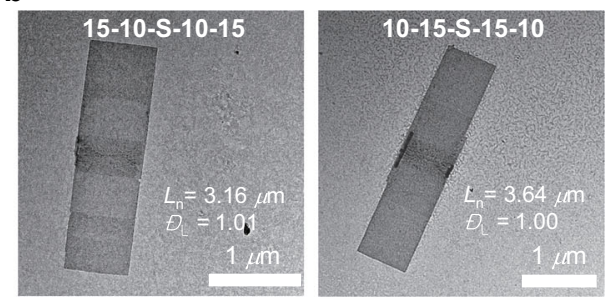

C
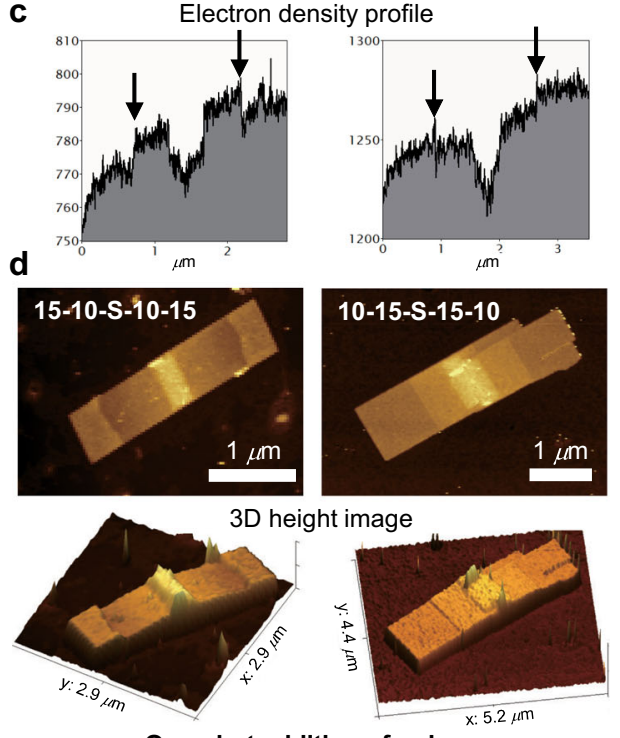

e
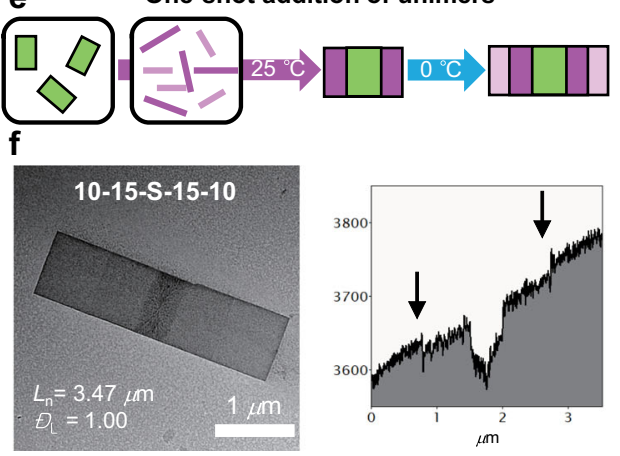

Fig. 5 Successful formation of symmetric penta-BCMs. a Schemes for the preparation of complex 2D BCMs by sequential addition of two unimers $\left(\mathrm{P} 2_{10}\right.$ and $\left.\mathrm{P} 2_{15}\right)$. By changing the order of the unimer addition, two types of symmetric penta-BCMs were obtained. The $2 \mathrm{D}$ nanosheets of longer $\mathrm{P} 2_{15}$ unimers are shown in dark pink, and those of $\mathrm{P}_{10}$ unimers are shown in light pink. b TEM images, c electron density profiles, and $\mathbf{d} 2 \mathrm{D}$, and 3D height AFM images of the penta-BCMs showing clear distinctions of the two types of penta$\mathrm{BCMs}$ prepared by the sequential addition. e Scheme for the preparation of one $2 \mathrm{D} \mathrm{BCM}$ by one-shot addition of two unimers. $\mathrm{f}$ TEM image and electron density profile of the penta-BCM obtained by one-shot living CDSA. Numbers in the images indicate the average length $\left(L_{n}\right)$ and dispersity $\left(\Theta_{L}\right)$.

Supplementary Figs. 28, 29). A clear distinction in contrast is observed in the TEM images as the A block of $\mathrm{P} 2{ }_{15}$ appears darker due to its higher electron density than the B block of $\mathrm{P} 2_{10}$ (Fig. 5c). Furthermore, AFM analysis also confirms the blocky structure of penta-BCMs, showing another clear difference in the height of 2D sheets (Fig. 5d and Supplementary Fig. 30). We even attempted a more challenging but simple one-shot BCM formation by adding two $\mathrm{P} 2$ unimers to $2 \mathrm{D}$ seeds at the same time with each U/S ratio of 3 at $25^{\circ} \mathrm{C}^{50}$. Since more crystalline $\mathrm{P} 2_{15}$ grew much faster than $\mathrm{P} 2_{10}$, the longer $\mathrm{P} 2_{15}$ preferentially assembled on the $2 \mathrm{D}$ seeds. Then, lowering the temperature to $0{ }^{\circ} \mathrm{C}$ initiated the self-assembly of the shorter $\mathrm{P} 2_{10}$ via the seeded-growth mechanism, resulting in the formation of the same B-A-S-A-B penta-BCM as that prepared by sequential addition (Fig. 5e, Supplementary Figs. 30, 31). This result demonstrates an excellent example of this new strategy that could be used to construct complex nanostructures based on a deep understanding of the $2 \mathrm{D}$ assembly process.

Since these precisely controlled $2 \mathrm{D}$ rectangles were composed of fluorescent conjugated PCPVs, they were visible under super-resolution structured illumination microscopy (SR-SIM) without additional dye. Interestingly, 2D seeds in the middle showed much higher fluorescence than the rest of the $2 \mathrm{D}$ sheets freshly formed from P2 unimers, indicating that the longer P2 emits stronger light (Supplementary Fig. 32). In addition, a video of the micelle solution recorded by confocal laser scanning microscopy (CLSM) shows a persistent shape and fluorescence stability without decomposition and photobleaching (Supplementary Video 1).

\section{Discussion}

In conclusion, we successfully demonstrated the formation of uniform semi-conducting $2 \mathrm{D}$ rectangles having sharp edges from a semi-crystalline conjugated homopolymer by the uniaxial seeded-growth approach. This intriguing direction-selective assembly allowed us to control the length of $2 \mathrm{D}$ rectangles for the first time with narrow dispersity through 2D CDSA. Taking advantage of this uniaxial growth from a homopolymer, 2D growth kinetic studies revealed that the homopolymer selfassembly followed ideal first-order kinetics, similar to living polymerization. This result indicates that the polymer selfassembly follows ideal crystallization because disturbing elements, such as the conformational effect of a shell block or back-bone chain folding, were eliminated. Lastly, 2D CDSA produced several complex but well-controlled penta-BCMs using various sizes of P2. Ultimately, we succeeded in a one-shot BCM formation based on an understanding of the growth kinetics, which provided an excellent guideline for optimizing self-assembly conditions. These precisely controlled uniform fluorescent 2D nanostructures would have great potential for optoelectronic applications.

\section{Methods}

Polymerization procedure. A $4 \mathrm{~mL}$ sized screw-cap vial with septum was flame dried and charged with a monomer and a magnetic bar. The vial was purged with $\operatorname{Ar}_{(\mathrm{g})}$ three times, and degassed anhydrous THF was added $\left([\mathrm{M} 1]_{0}=0.5 \mathrm{M}\right.$ or $\left.[\mathrm{M} 2]_{0}=0.1 \mathrm{M}\right)$. After the $\mathrm{Ar}_{(\mathrm{g})}$-purged G3 catalyst in the other $4 \mathrm{~mL}$ vial was dissolved in $50 \mu \mathrm{L}$ THF, the solution was rapidly injected to the monomer solution at $0^{\circ} \mathrm{C}$ under vigorous stirring. After the complete conversion of M1 to P1 (or M2 to $\mathrm{P} 2$ for $\mathrm{P} 2$ homopolymer), for the block copolymer (BCP) formation, the second monomer $(\mathrm{M} 2)$ was added $\left([\mathrm{M} 2]_{0}=0.1 \mathrm{M}\right)$ to the vial at $0{ }^{\circ} \mathrm{C}$. The reaction was quenched by excess ethyl vinyl ether (EVE) after the desired reaction time and precipitated in methanol at room temperature. The obtained purple solid was filtered and dried in vacuo. The monomer conversions were calculated from the ${ }^{1} \mathrm{H}$ NMR spectra of the remained crude mixture. Both P1-b-P2 BCP and P2 homopolymer have been characterized separately in the reference ${ }^{42,44}$.

Preparation of blends of $\mathbf{P 1}_{\mathbf{5 0}}-\boldsymbol{b}-\mathbf{P} \mathbf{2}_{\mathbf{2 2}}$ and $\mathbf{P} \mathbf{2}_{\mathbf{2 2}}$. Each polymer $\left(\mathrm{P}_{50}-b-\mathrm{P} 2_{22}\right.$ and $\mathrm{P} 2_{22}$ ) was dissolved in $0.5 \mathrm{~g} / \mathrm{L}$ chloroform (A total volume of blends was more than $0.5 \mathrm{~mL}$ in $4 \mathrm{~mL}$ vial). Without aging, two solutions were mixed at room temp. with various $\mathrm{P}_{50^{-}}-b-\mathrm{P} 2_{22}$ and $\mathrm{P} 2_{22}$ with a ratio of 2:1 (or molar ratio of 1:2). 
Preparation of 2D seeds from the blend by heating and aging. The blended solutions were sealed with a Teflon lined cap and heated at $50^{\circ} \mathrm{C}$ for $1 \mathrm{~h}$, followed by cooling down to $25^{\circ} \mathrm{C}$ and aging for 3 days. The resulting 2D nanoparticles were observed by AFM and TEM imaging. In details, samples for TEM and AFM imaging were prepared by spin-coating one drop $(c a .10 \mathrm{~mL})$ of the $2 \mathrm{D}$ seeds colloidal solution onto a carbon-coated copper grid (for TEM imaging) or freshly cleaved mica (for AFM imaging) (spinning rate $=3000 \mathrm{rpm}$ for $30 \mathrm{secs}$ ). Devicerelated information is described in the Supplementary Information.

Preparation of 2D rectangles with tunable length via living 2D CDSA of $\mathbf{P 2}_{\mathbf{n}}$ unimers. After diluting the $2 \mathrm{D}$ seeds solution to $0.03 \mathrm{~g} / \mathrm{L}$ chloroform, a solution of lower molecular weight $\mathrm{P} 2_{\mathrm{n}}$ (unimer, $M_{\mathrm{n}}=5.0-6.1 \mathrm{kDa}, Ð<1.18$ ) in $10 \mathrm{~g} / \mathrm{L}$ chloroform was added to the solution of the $2 \mathrm{D}$ seeds with various unimer-to-seed $(\mathrm{U} / \mathrm{S})$ mass ratios. Then the samples were aged at $\mathrm{X}^{\circ} \mathrm{C}$ (variable temperatures).

Growth kinetic studies of the living 2D CDSA. To conduct growth kinetic studies of the $2 \mathrm{D}$ assembly in solution, we monitored the living 2D assembly over aging time after adding the unimer solution to the seed solution. For each nanostructure, length, width, height, area, aspect ratio, and angle distributions were estimated from the TEM and AFM images manually using the ImageJ software package, which developed at the US National Institute of Health. For the statistical length analyses, more than 30 randomly picked objects were processed to determine the average values depending on the dataset. Every particle in each image was counted to reduce subjectivity.

\section{Data availability}

The data that support the findings of this study, including the Supplementary Information, are available from the corresponding author upon request.

Received: 8 December 2020; Accepted: 1 April 2021;

Published online: 10 May 2021

\section{References}

1. Zhang, X. \& Xie, Y. Recent advances in free-standing two-dimensional crystals with atomic thickness: design, assembly and transfer strategies. Chem. Soc. Rev. 42, 8187-8199, https://doi.org/10.1039/C3CS60138B (2013).

2. Low, J., Cao, S., Yu, J. \& Wageh, S. Two-dimensional layered composite photocatalysts. Chem. Commun. 50, 10768-10777 (2014).

3. Zeng, M., Xiao, Y., Liu, J., Yang, K. \& Fu, L. Exploring two-dimensional materials toward the next-generation circuits: from monomer design to assembly control. Chem. Rev. 118, 6236-6296 (2018).

4. Wang, Z. et al. Two-dimensional light-emitting materials: preparation, properties and applications. Chem. Soc. Rev. 47, 6128-6174 (2018).

5. Wakahara, T. et al. Fullerene/cobalt porphyrin hybrid nanosheets with ambipolar charge transporting characteristics. J. Am. Chem. Soc. 134, 7204-7206 (2012).

6. Liu, K., Wang, L. \& Dong, R. Two-dimensional conjugated polymer films via liquid-interface-assisted synthesis toward organic electronic devices. J. Mater. Chem. C. 8, 10696-10718 (2020).

7. Zhang, X.-L., Wang, L., Chen, L., Ma, X.-Y. \& Xu, H.-X. Ultrathin 2D conjugated polymer nanosheets for solar fuel generation. Chi. J. Polym. Sci. 37, 101-114 (2019).

8. Qin, J. et al. Controlled growth of a large-size 2D selenium nanosheet and its electronic and optoelectronic applications. ACS Nano 11, 10222-10229 (2017).

9. Qiu, H. et al. Uniform patchy and hollow rectangular platelet micelles from crystallizable polymer blends. Science 352, 697 (2016)

10. He, X. et al. Two-dimensional assemblies from crystallizable homopolymers with charged termini. Nat. Mater. 16, 481-488 (2017).

11. Ganda, S. et al. Two-dimensional self-assembled structures of highly ordered bioactive crystalline-based block copolymers. Macromolecules 50, 8544-8553 (2017).

12. Inam, $M$. et al. $1 \mathrm{D}$ vs. $2 \mathrm{D}$ shape selectivity in the crystallization-driven selfassembly of polylactide block copolymers. Chem. Sci. 8, 4223-4230 (2017)

13. He, X. et al. Complex and hierarchical $2 \mathrm{D}$ assemblies via crystallization-driven self-assembly of poly(l-lactide) homopolymers with charged termini. J. Am. Chem. Soc. 139, 9221-9228 (2017).

14. Han, L. et al. Uniform two-dimensional square assemblies from conjugated block copolymers driven by $\pi-\pi$ interactions with controllable sizes. Nat. Commun. 9, 865 (2018).

15. Zhu, Y. et al. Three-dimensional spirals of conjugated block copolymers driven by screw dislocation. Macromolecules 53, 3217-3223 (2020).
16. Hudson, Z. M. et al. Tailored hierarchical micelle architectures using living crystallization-driven self-assembly in two dimensions. Nat. Chem. 6, 893-898 (2014).

17. Inam, M. et al. Controlling the size of two-dimensional polymer platelets for water-in-water emulsifiers. ACS Cent. Sci. 4, 63-70 (2018).

18. Wang, J., Zhu, W., Peng, B. \& Chen, Y. A facile way to prepare crystalline platelets of block copolymers by crystallization-driven self-assembly. Polymer 54, 6760-6767 (2013).

19. Osichow, A. et al. Ideal polyethylene nanocrystals. J. Am. Chem. Soc. 135, 11645-11650 (2013)

20. Zheng, Y. et al. Supramolecular thiophene nanosheets. Angew. Chem. Int. Ed. 52, 4845-4848 (2013).

21. Qi, R., Zhu, Y., Han, L., Wang, M. \& He, F. Rectangular platelet micelles with controlled aspect ratio by hierarchical self-assembly of poly(3-hexylthiophene)b-poly(ethylene glycol). Macromolecules 53, 6555-6565 (2020).

22. Lin, C.-H., Tung, Y.-C., Ruokolainen, J., Mezzenga, R. \& Chen, W.-C. Poly [2,7-(9,9-dihexylfluorene)]-block-poly(2-vinylpyridine) Rod-Coil and Coil -Rod-Coil Block Copolymers: synthesis, morphology and photophysical properties in methanol/THF mixed solvents. Macromolecules 41, 8759-8769 (2008).

23. Yu, Z. et al. Self-assembly of well-defined poly(3-hexylthiophene) nanostructures toward the structure-property relationship determination of polymer solar cells. J. Phys. Chem. C. 116, 23858-23863 (2012).

24. $\mathrm{Li}, \mathrm{X}$. et al. Uniform electroactive fibre-like micelle nanowires for organic electronics. Nat. Commun. 8, 15909 (2017).

25. Jin, X.-H. et al. Long-range exciton transport in conjugated polymer nanofibers prepared by seeded growth. Science 360, 897 (2018).

26. Schmelz, J., Karg, M., Hellweg, T. \& Schmalz, H. General pathway toward crystalline-core micelles with tunable morphology and corona segregation. ACS Nano 5, 9523-9534 (2011).

27. Yu, B., Jiang, X. \& Yin, J. Size-tunable nanosheets by the crystallization-driven 2D self-assembly of hyperbranched poly(ether amine) (hPEA). Macromolecules 47, 4761-4768 (2014).

28. Song, S. et al. Solvent effects leading to a variety of different $2 \mathrm{D}$ structures in the self-assembly of a crystalline-coil block copolymer with an amphiphilic corona-forming block. Chem. Sci. 11, 4631-4643 (2020).

29. Jarrett-Wilkins, C. N. et al. Linear and branched fiber-like micelles from the crystallization-driven self-assembly of heterobimetallic block copolymer polyelectrolyte/surfactant complexes. Macromolecules 52, 7289-7300 (2019).

30. Xu, J., Zhou, H., Yu, Q., Manners, I. \& Winnik, M. A. Competitive selfassembly kinetics as a route to control the morphology of core-crystalline cylindrical micelles. J. Am. Chem. Soc. 140, 2619-2628 (2018)

31. Douglas, F. J. et al. Self-assembly of ultra-thin lanthanide oxide nanowires via surfactant-mediated imperfect oriented attachment of nanoparticles. Cryst. Eng. Comm. 14, 7110-7114 (2012).

32. Radovsky, G., Shalev, T. \& Ismach, A. Tuning the morphology and chemical composition of MoS2 nanostructures. J. Mater. Sci. 54, 7768-7779 (2019).

33. Pearce, S. et al. Uniform, high-aspect-ratio, and patchy $2 \mathrm{D}$ platelets by living crystallization-driven self-assembly of crystallizable poly (ferrocenyldimethylsilane)-based homopolymers with hydrophilic charged termini. Macromolecules 52, 6068-6079 (2019).

34. $\mathrm{Hu}, \mathrm{W}$. Growth rate equations of lamellar polymer crystals. Polym. Crystal. 1 , e25838 (2018)

35. Majumder, S., Busch, H., Poudel, P., Mecking, S. \& Reiter, G. Growth kinetics of stacks of lamellar polymer crystals. Macromolecules 51, 8738-8745 (2018).

36. Van Horn, R. M., Steffen, M. R. \& O'Connor, D. Recent progress in block copolymer crystallization. Polym. Crystal. 1, e10039 (2018).

37. Boott, C. E. et al. Probing the growth kinetics for the formation of uniform $1 \mathrm{D}$ block copolymer nanoparticles by living crystallization-driven self-assembly. ACS Nano 12, 8920-8933 (2018).

38. Ogi, S., Sugiyasu, K., Manna, S., Samitsu, S. \& Takeuchi, M. Living supramolecular polymerization realized through a biomimetic approach. Nat. Chem. 6, 188-195 (2014).

39. Wehner, M. \& Würthner, F. Supramolecular polymerization through kinetic pathway control and living chain growth. Nat. Rev. Chem. 4, 38-53 (2020).

40. Mukhopadhyay, R. D. \& Ajayaghosh, A. Living supramolecular polymerization. Science 349, 241 (2015).

41. Fukui, T. et al. Seeded self-assembly of charge-terminated poly(3hexylthiophene) amphiphiles based on the energy landscape. J. Am. Chem. Soc. 142, 15038-15048 (2020)

42. Yang, S., Shin, S., Choi, I., Lee, J. \& Choi, T. L. Direct formation of large-area 2D nanosheets from fluorescent semiconducting homopolymer with orthorhombic crystalline orientation. J. Am. Chem. Soc. 139, 3082-3088 (2017).

43. Yang, S., Kang, S. Y. \& Choi, T. L. Morphologically tunable square and rectangular nanosheets of a simple conjugated homopolymer by changing solvents. J. Am. Chem. Soc. 141, 19138-19143 (2019). 
44. Yang, S. \& Choi, T.-L. Rapid formation and real-time observation of micronsized conjugated nanofibers with tunable lengths and widths in 20 minutes by living crystallization-driven self-assembly. Chem. Sci. 11, 8416-8424 (2020).

45. Nazemi, A. et al. Uniform "Patchy" platelets by seeded heteroepitaxial growth of crystallizable polymer blends in two dimensions. J. Am. Chem. Soc. 139, 4409-4417 (2017).

46. Jeon, S. et al. Reversible disorder-order transitions in atomic crystal nucleation. Science 371, 498 (2021).

47. Jiang, X., Reiter, G. \& Hu, W. How chain-folding crystal growth determines the thermodynamic stability of polymer crystals. J. Phys. Chem. B 120, 566-571 (2016).

48. $\mathrm{Xu}$, J. et al. Synergistic self-seeding in one-dimension: a route to patchy and block comicelles with uniform and controllable length. Chem. Sci. 10, 2280-2284 (2019).

49. Li, X., Gao, Y., Harniman, R., Winnik, M. \& Manners, I. Hierarchical assembly of cylindrical block comicelles mediated by spatially confined hydrogenbonding interactions. J. Am. Chem. Soc. 138, 12902-12912 (2016).

50. Jin, B. et al. One-pot universal initiation-growth methods from a liquid crystalline block copolymer. Nat. Commun. 10, 2397 (2019).

\section{Acknowledgements}

This research was supported by the NRF, Korea through the following grants: Creative Research Initiative Grant and Postdoctoral Fellowship Program (Nurturing Nextgeneration Researchers, NRF grant no. 2020R1A6A3A01095653). We also thank the National Center for inter-University Research Facilities (NCIRF) at SNU for supporting CLSM and SR-SIM.

\section{Author contributions}

S.Y. and T.-L.C. conceived the project. S.Y. and S.-Y.K. carried out the experiments under the supervision of T.-L.C. All authors interpreted the data and discussed the experimental results.

\section{Competing interests}

The authors declare no competing interests.

\section{Additional information}

Supplementary information The online version contains supplementary material available at https://doi.org/10.1038/s41467-021-22879-6.

Correspondence and requests for materials should be addressed to T.-L.C.

Peer review information Nature Communications thanks Yiyong Mai and the other anonymous, reviewer(s) for their contribution to the peer review of this work. Peer reviewer reports are available.

Reprints and permission information is available at http://www.nature.com/reprints

Publisher's note Springer Nature remains neutral with regard to jurisdictional claims in published maps and institutional affiliations.

\begin{abstract}
Open Access This article is licensed under a Creative Common adtribution 4.0 International License, which permits use, sharing, appropriate credit to the original author(s) and the source, provide a link to the Creative Commons license, and indicate if changes were made. The images or other third party material in this article are included in the article's Creative Commons license, unless indicated otherwise in a credit line to the material. If material is not included in the article's Creative Commons license and your intended use is not permitted by statutory regulation or exceeds the permitted use, you will need to obtain permission directly from the copyright holder. To view a copy of this license, visit http://creativecommons.org/ licenses/by/4.0/
\end{abstract}

(C) The Author(s) 2021 